\title{
MRI with Arthroscopic Correlation in Meniscal Injuries of Knee
}

\author{
Rejeesh Saseendran ${ }^{1}$, Shameem Ahamed $\mathrm{M}^{2}$, Mohanan $\mathrm{K}^{3}$, Joy P Verghese ${ }^{4}$ \\ ${ }^{1}$ Assistant Professor, Department of Radiodiagnosis, ${ }^{2}$ Assistant Professor, Department of Radiodiagnosis, ${ }^{2}$ Professor and HOD, \\ Department of Radiodiagnosis, ${ }^{3}$ Associate Professor, Department of Radiodiagnosis, MES Medical College, Perinthalmanna, \\ Kerala India
}

Corresponding author: Dr. Shameem Ahamed M, Department of Radiodiagnosis, MES Medical College, Perinthalmanna, Kerala India.

DOI: 10.21276/ijcmsr.2018.3.2.17

How to cite this article: Rejeesh Saseendran, Shameem Ahamed M, Mohanan K, Joy P Verghese. MRI with arthroscopic correlation in meniscal injuries of knee. International Journal of Contemporary Medicine Surgery and Radiology. 2018;3(2):B70-B73.

\section{A B S T R A C T}

Introduction: Meniscal injuries are considered as the most common type of knee injury. Plain radiographs have only limited role in identification of soft tissue injuries. Advancement in arthroscopy and MRI have allowed for increased accuracy in diagnosing soft tissue injuries of the knee. Aim of the study was to correlate radiological and arthroscopic findings of meniscal injuries of knee.

Material and Methods: Cross sectional study was conducted in 40 patients who underwent MR imaging and arthroscopy following injury to the knee. The findings of MRI were correlated with the arthroscopy findings to find out the sensitivity, specificity, positive and negative predictive values.

Results: Out of 40 patients, $60 \%$ patients showed medial meniscal tears alone, 30\% patients showed lateral meniscal tears alone and $10 \%$ patients showed both medial and lateral meniscal tears on MRI. Sensitivity, specificity, positive predictive value (PPV) and negative predictive value (NPV) for detecting medial meniscal tears was $96.15 \%, 85 \% 92.8 \%$, and $91.4 \%$ respectively. In the detection of lateral meniscal tears the sensitivity, specificity, PPV and NPV were $88.2 \%, 95.6 \%, 93.7 \%$, and $91.66 \%$ respectively. Detection of lateral meniscus anterior horn tears, lateral meniscus body tears, bucket handle tears and root tears showed a higher sensitivity and specificity.

Conclusion: MRI has exclusive capability to assess the internal structure and the surface of the meniscus. It is more sensitive in detection of multiple meniscal tears that may be overlooked on arthroscopy. MRI should be considered in patients suspected to have meniscal injuries to prevent unwanted arthroscopies.

Key words: MRI, Arthroscopies, Meniscal Injuries, Medial Meniscal Tears, Lateral Meniscal Tears.

\section{INTRODUCTION}

Considerable improvement in knee imaging has been made in the area of MR imaging, which has apparently emerged as the primary tool, to guide the management of knee pain. With the development of new sequences, improved SNR (signal to noise ratio), higher resolution, reduced artefacts, shorter imaging times and improved accuracy, MRI has changed the traditional algorithm for workup of knee joint pathology, particularly in meniscal and cruciate ligamentous tears in knee injuries. ${ }^{1}$ With MRI injured knee can be visualized noninvasively, hence invasive procedures can avoid minimizing the further morbidity. MRI is now routinely used to assess a broad spectrum of internal knee derangements and articular disorders and has virtually replaced conventional arthrography in the evaluation of the menisci and cruciate ligaments and has decreased arthroscopic examinations. In addition to diagnostic benefits, MRI has also proved valuable in the selection of surgical candidates and preoperative planning. MRI provides superior anatomic and pathologic definition of soft tissue, ligaments fibrocartilage and articular cartilage. Additional advantages of MRI are multiplanar and thin section capabilities and the ability to evaluate subchondral bone and marrow. MRI, therefore, can pick up bone contusions and occult knee fractures, including tibial plateau fractures.

MRI is a non-invasive modality having high sensitivity, specificity and accuracy in the diagnosis of meniscal injuries. Many times arthroscopy is done without doing an MRI, only for diagnosis of ligament and meniscal injuries and may end up in negative results and is giving the patient an added surgical insult. ${ }^{2}$ Sometimes the more finer details of injuries may be missed by arthroscopy, which can be quickly picked up by MRI. An arthroscopy helps the orthopaedic surgeon to visualize the joint cavity with the aid of a camera introduced through a tiny skin incision. In case of the knee joint, arthroscopy helps in diagnosing meniscal and ligamentous tears and arthritis. The most significant advantage of arthroscopy is that the treating surgeon can intervene the problem. ${ }^{3}$ Exact diagnosis and appropriate treatment for knee related issues can be achieved by arthroscopic improvements by using high-resolution cameras.

Arthroscopy, in fact, is the direct visualization of surface 
morphology of various structures, but the more in-depth anatomy and morphology can be better made out with MRI only. Therefore in this respect, these modalities are complementary and supplementary. Hence the study was conducted to compare the MRI findings with arthroscopic findings and appearances of meniscal injuries of the knee and to find out the sensitivity, specificity, positive and negative predictive values of MRI in diagnosing meniscal injuries when compared with arthroscopy

\section{MATERIAL AND METHODS}

A cross-sectional study was conducted in Department of Radiodiagnosis, Government Medical College, Thrissur for a study period of 10 months. Total of 40 patients was selected who underwent MR imaging following an injury to the knee. Patients with knee injuries who underwent both MR imaging and arthroscopy of the knee, who were found to have a grade 3 meniscal injury with or without associated ligament injuries on MRI included in present study. Patients with neoplasms, inflammatory or infectious disorders or patients suffering a significant injury to the knee between the time of MRI and arthroscopy and patients with grade 1 or grade 2 intrameniscal signal changes were excluded from the study.

The MRI was done on the advice (Table 1) of the referring doctor. All eligible patients briefed about the procedure and gave informed consent before they entered into the study. History of their presenting complaint and details of any preexisting illness were asked. MRI scanning of the knee was performed using GE Signa HDxt machine with a 1.5 Tesla field strength magnet in a tightly coupled extremity. Findings of meniscal tears were classified into meniscus affected (medial or lateral), Area of involvement (anterior horn body or posterior horn), Type of tear (longitudinal, bucket handle, horizontal, radial tears, meniscal extrusion and root tear), Associated findings (Discoid meniscus, ACL or PCL tear, meniscal cyst, joint effusion).

Outcome measures: The various observations in MRI were recorded, and the report was written in a set pattern and was entered in the Performa for further analysis. The final diagnosis was made by the arthroscopic examination. All arthroscopy reports were reviewed. The findings of MRI were correlated with the Arthroscopy findings, which was taken as the gold standard.

\section{STATISTICAL ANALYSIS}

Descriptive analysis was used to calculate the sensitivity, specificity, positive predictive value (PPV), and the negative predictive value (NPV), in order to assess the reliability of clinical and MRI results.

\section{RESULTS}

Study group comprised of a total number of 40 patients, the age at presentation with knee pain ranged from 17 to 60 years. The mean age was 30 years, and the maximum number of patients affected belongs to the age group of 20-40 years. There were 27 (67.5\%) males, and 13(32.5\%) females in the patients included in the study.

Distribution of meniscal injuries: Of the 40 patients who were evaluated with MRI of the knee (Table-2) for evaluating painful knee joint following a trauma and found to have meniscal injuries, 24(60\%) showed medial meniscal tears alone,12(30\%) showed lateral meniscal tears alone and 4(10\%) both medial and lateral meniscal tears on MRI.

Distribution of medial meniscal tears: Only grade 3 tears were compared to grade1, and two tears do not reach up to

\begin{tabular}{|l|c|c|c|c|c|c|c|c|}
\hline Image & Sag T1 & Sag T2 & Sag PDFS & Cor T1 & AxPDFS & AxT1 & Cor & GRE \\
\hline Sequence & & & & & & & T2FS & \\
\hline TR & 750 & 2880 & 2280 & 960 & 4760 & 735 & 3040 & 350 \\
\hline TE & 12 & 70 & 36 & 12 & 32 & 12 & 70 & 14 \\
\hline PIXEL & $512 \times 192$ & $384 \times 224$ & $288 \times 160$ & $416 \times 224$ & $352 \times 192$ & $416 \times 192$ & $384 \times 224$ & $384 \times 224$ \\
\hline NEX & 2.00 & 4.00 & 4.00 & 4.00 & 2.00 & 3.00 & 4.00 & 2.00 \\
\hline FOV & $17 \times 16$ & $16 \times 16$ & $15 \times 15$ & $16 \times 16$ & $13 \times 13$ & $16 \times 16$ & $16 \times 16$ & $17 \times 17$ \\
\hline Sequence & FSE & FSE-XL & FSE -XL & FSE-XL & FSE-XL & FSE-XL & FSE-XL & FAST \\
\hline Time & $1.56 m i n$ & $2.47 m i n$ & $2.31 m i n$ & $2.43 m i n$ & $3.53 m i n$ & $2.39 m i n$ & $2.59 m i n$ & 3 min \\
\hline Thickness & 4 & 4 & 4 & 4 & 3 & 4.5 & 4 & 3.5 \\
\hline \multicolumn{7}{|c|}{ Table-1: Pulse sequences and imaging planes of MRI. } \\
\hline
\end{tabular}

\begin{tabular}{l|l|c|c|c|c|c|c|c|c|}
\hline & MM & LM & MMAT & MMBT & MMPT & LMAT & LMBT & LMPT \\
\hline MRI & 28 & 16 & 6 & 6 & 21 & 5 & 4 & 9 \\
\hline Arthroscopy & 27 & 17 & 6 & 6 & 20 & 4 & 4 & 10 \\
\hline True positive & 26 & 15 & 5 & 5 & 19 & 4 & 4 & 8 \\
\hline True negative & 11 & 22 & 33 & 33 & 18 & 35 & 3 & 29 \\
\hline False positive & 2 & 1 & 1 & 1 & 2 & 1 & 0 & 1 \\
\hline False negative & 1 & 2 & 1 & 1 & 1 & 0 & 0 & 2 \\
\hline $\begin{array}{l}\text { MM-medial meniscus, LM-lateral meniscus, MMAT-Medial meniscus anterior horn tears, MMBT- Medial meniscus body tears, } \\
\text { MMPT- Medial meniscus posterior horn tears, LMAT- Lateral meniscus anterior horn tears, LMBT- Lateral meniscus body tears, LMPT- } \\
\text { Lateral meniscus posterior horn tears }\end{array}$
\end{tabular}




\begin{tabular}{|l|c|c|c|c|c|c|c|}
\hline & H/O & LT & BHT & COM & RAD & RT & ACLI \\
\hline MRI & 11 & 11 & 4 & 5 & 3 & 2 & 18 \\
\hline Arthroscopy & 11 & 12 & 4 & 4 & 4 & 2 & 18 \\
\hline True positive & 9 & 10 & 4 & 4 & 3 & 2 & 17 \\
\hline True negative & 27 & 27 & 36 & 35 & 36 & 38 & 21 \\
\hline False positive & 2 & 1 & 0 & 1 & 0 & 0 & 1 \\
\hline False negative & 2 & 2 & 0 & 0 & 1 & 0 & 1 \\
\hline
\end{tabular}

H/O-horizontal/oblique tears. LT-longitudinal tear, BHT- Bucket handle tears, COM-complex tear, RAD-radial tear, RT- Root tears, ACLI$\mathrm{ACL}$ injuries

Table-3: Distribution of meniscal injuries

\begin{tabular}{|l|c|c|c|c|c|c|}
\hline & MM & LM & H/O & LT & RAD & COM \\
\hline Sensitivity(\%) & 96.29 & 88.2 & 81.8 & 83.3 & 75 & 100 \\
\hline Specificity(\%) & 85 & 95.6 & 93.1 & 96.5 & 100 & 97.2 \\
\hline PPV(\%) & 92.8 & 93.7 & 81.8 & 90.9 & 100 & 80 \\
\hline NPV(\%) & 91.4 & 91.66 & 93.1 & 93.1 & 97.2 & 100 \\
\hline MM-medial meniscus, LM-lateral meniscus, H/O-horizontal/oblique tears, LT-longitudinal tear, RAD-radial tear, COM-complex tear. \\
\hline \multicolumn{7}{|r|}{} \\
\hline
\end{tabular}

the articular surface and hence are invisible to arthroscopic surface evaluation. Out of 28 patients of medial meniscal tears, most common tear location was at the posterior horn. In our study, we found posterior horn tear in 21 patients (75\%), body tears in $6(21.4 \%)$ and anterior horn tears in 6 (21.4\%) patients on MRI and in arthroscopy. Out of the 21 Medial Meniscal posterior horn tears, there were two false positive and one false negative observation. Of 6 anterior horns and body tears 1 false negative and false positive result each were noted.

Distribution of lateral meniscal tears: Out of 16 patients with lateral meniscus tears, posterior horn tears were seen most commonly, in 9 (56.2\%). Anterior horn tears were seen in 5 patients (31.2\%), and tears involving the body were seen in $4(25 \%)$ patients. On Arthroscopy posterior horn tears were seen in 10 patients and anterior horn tears and meniscal body tears were seen in 4 patients. Of the 9 patients with lateral meniscal posterior horn tears who have been followed up by arthroscopy, there was one false positive and two false negative results. Among four patients with meniscal body tears, one false positive and no false negative result were there.

Distribution of meniscal injuries were explained in Table- 3. Eleven cases showed horizontal/oblique tear in both MRI and arthroscopy. There were two false positive and false negative results each. Eleven longitudinal tears were noted on MRI and 12 on arthroscopy, with one false positive and two false negative results.

In our study the sensitivity and specificity for detecting medial meniscal tears was $96.15 \%$ and $85 \%$ respectively. In the detection of Lateral Meniscal tears the sensitivity and specificity were $88.2 \%$ and $95.6 \%$ respectively. The sensitivity and specificity of MRI in identifying medial meniscal tear has been reported to range from $77 \%-97.5 \%$ and $37 \%-100 \%$ respectively.

\section{DISCUSSION}

MRI is a very useful non-invasive imaging modality having high sensitivity and specificity in the diagnosis of meniscal injuries. Meniscal injuries are most commonly seen in young adults and occur more in males. Most commonly injured site in the meniscus is the posterior horn. Our study reported a total of 28 medial and 16 lateral meniscal injuries, which is corresponding with La Prade et al., who reported that medial meniscal tears are twice more common than lateral meniscal tears. ${ }^{4}$ While finding the medial meniscal tears, our study shows some false positive and false negative reports. The exact cause of the false positives in detection of tears of the medial meniscus in our study was not apparent. It may be attributed to the presence of intra-meniscal tears not communicating with the articular surface of the meniscus or misinterpretation of normal anatomy or operator/procedure dependant drawback of arthroscopy. Study shows some false positive and false negative reports even while finding the lateral meniscal tears. Case which was falsely reported as positive on MRI was probably due to pseudo tear appearance of lateral meniscus caused by meniscofemoral ligament. We took a critical review of all the false positive meniscal tears reported on arthroscopy. However even on review study arthroscopic findings could not be correlated. Presence of ACL tear may have decreased the sensitivity of MRI in picking up the meniscal tears. Lee WH et al and De Smet AA et al have reported decreased sensitivity of MRI in identifying meniscal tears when an associated ACL injury was there. ${ }^{5,6}$

The false positive results while detecting horizontal/oblique tear may be due to misinterpretation of intrameniscal degenerative grade II signals for a tear. The false negative results were noted in cases with associated ACL tears, and indicate the reduced sensitivity of MRI in identification of meniscal tears in the setting of ACL injuries. Four patients were found to have radial tears of medial meniscus on MRI three of which three were confirmed on arthroscopy. All of them were noted on the posterior horn. Two case showed cleft sign and one showed abrupt truncation of inner part of meniscus. Harper KW et al have reported 37-89\% sensitivity for MRI in detecting radial tears. ${ }^{7}$ Five complex 
tears were noted on MRI, four of which were confirmed on arthroscopy. Three involved the medial meniscus and one, the lateral meniscus. Two of them had associated cruciate ligament injuries. The false positive result may be due to misinterpretation of intrameniscal grade II signal for a tear. In our study 4 patients showed bucket handle tears in medial meniscus all of which has been confirmed by subsequent arthroscopy. This is corresponding with study by Singson et al, who reported that medial meniscus bucket handle tears are more common. ${ }^{8}$ Of 4 patients with bucket handle tears, in 2 patients displaced fragment is seen in intercondylar notch, 3 patient showed double PCL sign and one patient showed absent bowtie sign. Eighteen patients had ACL injury on MRI and arthroscopy. There was one false positive and one false negative case each. Eight ACL tears were seen in patients with medial meniscal injury alone (33.3\%), 6 in patients with lateral meniscal injury alone (50\%) and 3(75\%) in patients with both medial and lateral meniscal injuries. This corresponds to studies by Jee WH et al and De Smet AA et al who reported that, in the setting of an acute ACL injury, the lateral meniscus is torn more commonly. ${ }^{9,10}$

In our study the sensitivity and specificity for detecting medial meniscal tears and lateral meniscal tears was found to be in accordance with Fischer et al. ${ }^{11}$ One patient had meniscal extrusion on MRI, which was confirmed by arthroscopy. Twenty eight patients had joint effusion at the time of MRI, and 24 at the time of arthroscopy. Two patients showed a meniscal cyst on MRI which were confirmed by arthroscopy. One was associated with a horizontal tear of posterior horn of medial meniscus and the other with a complex tear. PDFS, FSE T2 and GRE T2 sequences were found to be most sensitive to the spectrum of meniscal injuries. This corresponds to study done by Helms CA, who noted that FSE, PDFS and GRE sequences most useful in detection of meniscal injuries. ${ }^{12}$

\section{CONCLUSION}

MRI has high sensitivity and specificity in identifying bucket handle tear. Double PCL sign, a fragment in the notch sign, absent bow tie sign and truncated meniscus sign is the useful signs in detecting bucket handle tears. MRI has low sensitivity in identifying radial tears. The ghost sign, cleft sign, truncated triangle sign and marching cleft sign increases its sensitivity in identifying radial tears. Common causes of false positive findings in MRI is due to misinterpretation of intrameniscal signal changes (grade II signal changes) or normal anatomy as tears. Presence of associated ACL injury decreases the sensitivity of MRI in identifying meniscal tears. A discoid meniscus is a meniscal variant, more commonly seen in lateral meniscus and they are more prone to tears. Joint effusion is seen associated with meniscal and cruciate ligament injuries. PDFS, FSE T2 and GRE T2* sequences were found to be most sensitive to the spectrum of meniscal injuries. MRI is unique in its ability to evaluate the internal structure as well as the surface of the meniscus.MRI is advantageous in conditions where arthroscopy is not very useful like in inferior surface tears. It is more sensitive in detection of multiple meniscal tears that may be overlooked on arthroscopy. MRI is more sensitive than arthroscopy in the detection of grade I and II intrasubstance degeneration. Since MRI is non-invasive, it does not involve morbidity associated with arthroscopy. MRI should be considered in patients suspected to have meniscal injuries to prevent unwanted arthroscopies.

\section{ACKNOWLEDGEMENT}

Authors are thankful to Mr Jaleel, Mrs Rajitha and Medi research direct for their support during entire project.

\section{REFERENCE}

1. Singh JP, Garg L, Shrimali R, Setia V, Gupta V. MR Imaging of knee with arthroscopic correlation in twisting injuries. Indian journal of radiology and imaging. 2004;14(1):33.

2. McIlwraith CW, Wright I, Nixon AJ. Diagnostic and Surgical Arthroscopy in the Horse-E-Book. Elsevier Health Sciences; 2014 Jun 27.

3. Kelly BT, Williams RJ, Philippon MJ. Hip arthroscopy: current indications, treatment options, and management issues. The American journal of sports medicine. 2003;31(6):1020-37.

4. La Prade RF, Burnett QM, Veenstra MA, Hodgman CG. The prevalence of abnormal MRI findings in asymptomatic knees. Am J Sp Med 1994; 171(2): 761766.

5. Lee JH, Singh TT, Bolton G. Axial fat-saturated FSE imaging of knee: appearance of meniscal tears. Skeletal Radiol 2002;31(7):384-95.

6. De Smet AA, Wright DH, Norris M. Buckethandletears of the medial and lateral menisci of the knee: value of MR imaging in detecting displaced fragments. AJR Am J Roentgenol 1995;165(3):621-5.

7. Harper KW, Helms CA, Lambert HS 3rd, et al.Radial meniscal tears: significance,incidence, and MR appearance. AJR Am J Roentgenol 2005;185(6):1429_ 34

8. Singson RD, Feldman F, Staron R, Kiernan H. MR imaging of the displaced bucket handle tear of the medial meniscus. AJR 1991; 156(6): 121.

9. Jee WH, McCauley TR, Kim JM. Magnetic resonance diagnosis of meniscal tears in patients with acute anterior cruciate ligament tears. J Comput Assist Tomogr 2004;28(3):402-6.

10. De Smet AA, Graf BK. Meniscal tears missed on MR imaging: relationship to meniscal tear patterns and anterior cruciate ligament tears. AJR Am J Roentgenol 1994;162(4):905-11.

11. Fischer SP, Fox JM, Del Pizzo W et al . Accuracy of diagnosis from magnetic resonance imaging of the knee: a multi centre analysis of one thousand and fourteen patients. J Bone Joint Surg (AM) 1991; 73(4): 2-10

12. Helms CA. The meniscus: recent advances in MR imaging of the knee. AJR Am J oentgenol 2002;179(5):1115-22.

Source of Support: Nil; Conflict of Interest: None

Submitted: 18-02-2018; Accepted: 22-03-2018; Published online: 19-05-2018 